\title{
Factores de riesgo asociados a la hipoglicemia en neonatos de riesgo
}

\section{Risk factors associated with hypoglycemia in neonates}

\author{
Chantal Marie Real Aparicio ${ }^{(1)}$, Patricia Arias Yrazusta ${ }^{(1)}$
}

\section{RESUMEN}

Introducción: La hipoglicemia es común en el recién nacido $(\mathrm{RN})$ pero si es persistente o muy profunda puede generar secuelas neurológicas. Los objetivos de esta investigación fueron describir los valores de glicemia capilar de los recién nacidos de riesgo y determinar los factores de riesgo de hipoglicemia de los neonatos de riesgo del Hospital Nacional (Itauguá, Paraguay). Materiales y Métodos: Se midió la glicemia capilar con glucómetro digital en los RN de riesgo Hospital Nacional (Itauguá, Paraguay) entre mayo 2015 y febrero 2016. Para determinar los factores de riesgo se aplicó luego un diseño de casos (RN con hipoglicemia) y controles (RN sin hipoglicemia). Las variables independientes fueron las patologías asociadas del $\mathrm{RN}$, prematurez, restricción del crecimiento intrauterino, parto por cesárea, sexo del RN, hipertensión arterial y diabetes gestacional. Resultados: Se incluyeron $76 \mathrm{RN}, 56,6 \%$ del sexo masculino, con edad gestacional media $36 \pm 3$ semanas, con mediana de peso $2.569 \mathrm{~g}$, nacidos por cesárea en $68,4 \%$. Se encontraron 25 $\mathrm{RN}$ con hipoglicemia $<47 \mathrm{mg} / \mathrm{dL}$ (casos) y $51 \mathrm{sin}$ hipoglicemia (controles). Los factores de riesgo estadísticamente significativos asociados a la hipoglicemia neonatal fueron la prematurez y la presencia de patologías asociadas: dificultad respiratoria, sepsis y asfixia perinatal. Se halló considerable correlación entre glicemia capilar y glicemia plasmática $(r+0,6)$. Conclusiones: Los factores de riesgo estadísticamente significativos asociados a la hipoglicemia neonatal fueron la presencia de patologías asociadas (dificultad respiratoria, sepsis y asfixia perinatal) y la prematurez. La correlación entre la glicemia capilar y la plasmática fue considerable $(r+0,6)$.

Palabras clave: Hipoglicemia, Recién nacido, prematurez, glucómetro, glucosa.

\begin{abstract}
Introduction: Hypoglycemia is common in the newborn (RN) but if it is persistent or too deep can cause neurological sequelae. The objectives of this study were to describe the values determined by capillary glycemia digital glucometer in the $\mathrm{RN}$ risk and analyze the risk factors associated with hypoglycemia. Materials and Methods: Capillary glucose was measured with digital glucometer in the risk RN National Hospital (Itauguá, Paraguay) between May 2015 and February 2016. To determine the risk factors design cases ( $R N$ with hypoglycemia) was then applied and controls (RN without hypoglycemia). The independent variables were associated pathologies $\mathrm{RN}$, prematurity, intrauterine growth restriction, cesarean birth, sex $\mathrm{RN}$, high blood pressure and gestational diabetes. Results: RN 76 were included, $56.6 \%$ male, mean age $36 \pm 3$ gestational weeks with median weight $2,569 \mathrm{~g}$, born by Caesarean section at $68.4 \%$. RN 25 were found with hypoglycemia $<47 \mathrm{mg} / \mathrm{dL}$ (cases) and 51 without hypoglycemia (controls). Statistically significant risk factors associated with neonatal hypoglycemia were prematurity and the presence of associated diseases: respiratory distress, sepsis and perinatal asphyxia. It was found significant correlation between capillary glycemia and plasma glucose $(r+0.6)$. Conclusions: Significant risk factors statistically associated with neonatal hypoglycemia were the presence of associated diseases (respiratory distress, sepsis and perinatal asphyxia) and prematurity. The correlation between capillary glucose and plasma was significant $(r+0.6)$.
\end{abstract}

Keywords: Hypoglycemia, Newborn, prematurity, blood glucose meter, glucose.

1. Postgrado en Pediatría Universidad Católica Nuestra Señora de la Asunción, Departamento de Pediatría del Hospital Nacional de Itauguá. Paraguay.

Correspondencia: Dra. Chantal Marie Real Aparicio. E-mail: chanti_real@hotmail.com

Conflicto de intereses: Los autores declaran no poseer conflicto de interés.

Recibido: 12/07/2016. Aceptado: 12/12/2016.

Doi: 10.18004/ped.2016.diciembre.213-219 


\section{INTRODUCCIÓN}

Las concentraciones bajas de glucemia son frecuentes en recién nacidos (RN) sanos de 1 a 2 horas después del nacimiento, sobre todo por el cese del aporte materno por la placenta y problemas en la alimentación oral ${ }^{(1)}$. Muchos neonatos compensan esa "hipoglicemia fisiológica" con la producción de cuerpos cetónicos derivados de las grasas, como fuente de energía ${ }^{(2)}$. Pero si la hipoglicemia es persistente o recurrente y el $\mathrm{RN}$ presenta otros factores de riesgo, este imbalance del metabolismo glucídico puede generar secuelas neurológicas ${ }^{(3,4)}$.

En el año 2011 el Comité del feto y recién nacido de la Academia Americana de Pediatría no encontró evidencia científica para discriminar entre glicemia normal y baja en el RN de pretérmino y de término aunque propuso como límite inferior aceptable o normal un valor de $47 \mathrm{mg} / \mathrm{dL}$ (2,6 mmol/L) y un nivel de $45 \mathrm{mg} / \mathrm{dL}$ (2,5 mmol/L) como límite de glicemia para actuar. Sí puso énfasis en identificar a los RN en riesgo de desarrollarla y las medidas profilácticas a $\operatorname{aplicar}^{(3,5-7)}$.

La hipoglicemia neonatal $<45 \mathrm{mg} / \mathrm{dL}$ ocurre en 5 a $7 \%$ de los RN, pero puede variar entre valores de 3,2 a $14,7 \%$ en RN de pretérmino ${ }^{(7-9)}$.

En base a las etiologías de la hipoglucemia neonatal, se han establecido los grupos de riesgo: prematurez, macrosomía, madre con diabetes gestacional, vía de parto, restricción del crecimiento intrauterino (RCIU), ingreso a terapia intensiva, policitemia, hipoxia perinatal ${ }^{(8,9)}$.

Los signos clínicos de hipoglicemia no son específicos e incluyen manifestaciones que son comunes a neonatos afectos de diversas patologías: apatía, ligera hipotonía, irritabilidad, llanto anormal, temblores, pobre succión y alimentación, vómitos, respiración irregular, taquipnea, apneas, cianosis, convulsiones, estupor, coma, hipotermia, llanto débil ${ }^{(10)}$.

El gold estándar del diagnóstico de hipoglicemia es la determinación de la glucemia en plasma con cualquiera de las determinaciones laboratoriales: glucosa oxidasa, hexokinasa o el método de la deshidrogenasa ${ }^{(2,411)}$. La desventaja de esta medición laboratorial es el relativo retraso en la obtención del resultado y el consumo por los eritrocitos cuando se retrasa el análisis. Por ello, se han desarrollado los métodos rápidos en tiras reactivas y lecturas con glucómetros $^{(2)}$. La correlación entre estos métodos en plasma y en sangre total es buena aunque puede variar en 10 a $20 \mathrm{mg} / \mathrm{dL}$. Lastimosamente, esta diferencia se acrecienta con niveles bajos de glucemia ${ }^{(2)}$. La medición de la glicemia capilar es una técnica intermitente, invasiva y dolorosa, por lo que es un desafío que puede llevar a exageraciones en el tratamiento o dificultad para la detección precoz de hipoglicemias ${ }^{(8,12)}$.

La detección de la glucemia capilar con glucómetros en la cabecera del paciente tiene variada sensibilidad y especificidad, en relación a las marcas comerciales ${ }^{(13)}$. Así, la sensibilidad oscila entre 92 y $96 \%$, con valor predictivo negativo entre 90 y $96 \%{ }^{(13)}$. Por ello, se recomienda la medición con varios equipos y la confirmación con la técnica habitual en plasma en laboratorio ${ }^{(13)}$. Este defecto de los glucómetros se debe a que fueron diseñados para adultos y sobre todo para detectar hiperglucemias. Por ello, la Asociación Americana de Diabetes ha fijado que los glucómetros tengan un error $<10 \%$ con respecto a la medición en laboratorio con rangos de glucemia entre 30 y $400 \mathrm{mg} / \mathrm{dL}^{(13,14)}$. La medición de la glucemia varía además con la hemoglobina de los $\mathrm{RN}$ : la anemia genera falsas hiperglicemias y la poliglobulia baja las lecturas de los glucómetros.

Si bien los glucómetros son fáciles de usar, sus resultados son influenciados por múltiples factores: variaciones interobservador, hematocrito, hiperbilirrubinemia, altitud, temperatura, humedad ambiental, hipotensión arterial, presión de $\mathrm{O}_{2}$ e hipertrigliceridemia. Además, debe observarse la especificación técnica de cada marca pues algunas requieren sangre venosa, arterial o capilar exclusivamente $^{(14,15)}$.

De forma general en todos los grupos con riesgo de desarrollar hipoglucemia debe realizarse medición de glucosa en la primera hora de vida y posteriormente cada 2 horas en las primeras 8 horas de vida y cada 4-6 horas en las siguientes 24 horas de vida. El diagnóstico bioquímico y clínico se confirma por la desaparición inmediata de los síntomas tras la administración de glucosa y la corrección de la hipoglucemia ${ }^{(7)}$. 
La Organización Mundial de la salud recomienda como tratamiento que los $\mathrm{RN}$ de riesgo deben ser alimentados a la 1 hora de vida y evaluados luego de 30 minutos. El monitoreo debe mantenerse por $12 \mathrm{hs}$ en RN de madres diabéticas y en fetos macrosómicos. Pero el monitoreo debe ser por 24 hs en aquellos $\mathrm{RN}$ de pretérmino o pequeños para su edad gestacional ${ }^{(2)}$. Si se documenta inadecuada homeostasis de la glucosa postnatal, el Pediatra debe estar seguro de que el $\mathrm{RN}$ puede mantener concentraciones normales de glucosa en plasma ${ }^{(16-21)}$.

La hipoglucemia sigue siendo un problema en Neonatología porque persisten las controversias sobre su definición, importancia clínica y manejo clínico óptimo $^{(22-24)}$. La evidencia actual no especifica una glucemia que se considere normal o anormal, tampoco aquel valor que sea potencialmente dañino para el sistema nervioso ${ }^{(25)}$. Una solución pragmática es la identificación precoz de $\mathrm{RN}$ en riesgo de desarrollar hipoglicemia y la instalación de medidas preventivas ante la falta de una definición clara de hipoglicemia neonatal ${ }^{(2)}$.

Los objetivos de esta investigación fueron describir los valores de glicemia capilar de los recién nacidos de riesgo y determinar los factores de riesgo de hipoglicemia de los neonatos de riesgo del Hospital Nacional (Itauguá, Paraguay). Además, describir las características demográficas y clínicas de los recién nacidos de riesgo, determinar los síntomas asociados a la hipoglicemia del recién nacido y finalmente correlacionar la glicemia capilar con la plasmática.

\section{MATERIALES Y MÉTODOS}

Se aplicó un diseño de casos y controles. Se consideraron casos a los neonatos de sexo femenino y masculino, con hipoglicemia en al menos una medición, nacidos en el Hospital Nacional desde mayo del 2015 hasta febrero 2016. Actuaron como controles los neonatos de sexo femenino y masculino, sin hipoglicemia en las mediciones, nacidos en el mismo periodo de tiempo del mismo hospital.

Los criterios de inclusión fueron:

1. Recién nacidos de riesgo: prematurez, macrosomía, neonato grande para la edad gestacional, hijo de madre diabética, restricción del crecimiento intrauterino, bajo peso al nacer, hipoxia perinatal.

2. Nacimientos por cesárea o parto vaginal.
3. Controles de glicemia capilar a partir de los 30 minutos de vida y al menos 3 controles en las primeras 24 horas.

Los criterios de exclusión fueron:

1. Malformaciones congénitas detectadas por ecografía prenatal o en sala de partos.

2. Hoja de nacimiento con datos incompletos.

3. Nacimiento en otro servicio

La variable dependiente fue la hipoglicemia y las independientes fueron las patologías asociadas del RN (dificultad respiratoria, sepsis y asfixia perinatal), prematurez, restricción del crecimiento intrauterino, parto por cesárea, sexo del $\mathrm{RN}$, hipertensión arterial y diabetes gestacional.

Para calcular el tamaño de muestra se utilizó el programa estadístico Epi Info $7^{\circledR}$ considerando el ser hijo de madre diabética como el principal factor de riesgo de hipoglicemia en el recién nacido. Para una proporción de $51 \%$ de hipoglicemia en hijos de madres diabéticas y 5\% de hipoglicemia en hijos de madres no diabéticas ${ }^{9}$, error alfa $5 \%$, error beta $20 \%$, razón 1:2 entre casos y controles, IC 95\%, el tamaño mínimo calculado fue de 13 casos y 25 controles.

Las fuentes de información/recolección fueron la hoja de nacimiento y hoja de enfermería de los pacientes con factores de riesgo para hipoglicemia que nacieron en el Servicio de Nursery y que fueron sometidos a determinación de glicemia capilar por el personal médico y de enfermería del servicio.

Para asegurar el control de calidad, las glicemias fueron medidas con un solo equipo de la marca $\mathrm{OnCall}^{\oplus}$, que fue calibrado periódicamente. Además, se realizó un pretest con 10 sujetos para detectar errores y rectificarlos.

Las variables fueron procesadas con el programa estadístico Epi Info $7^{\circledast}$ utilizando las pruebas ANOVA, chi ${ }^{2}$ y Kruskall Wallis. Se consideró significativa toda $\mathrm{p}<0,01$.

Se respetaron los Principios de la Bioética. Esta investigación no requirió consentimiento informado pues se trabajó con expedientes médicos. Los casos de hipoglicemia fueron tratados según protocolo del Servicio. La Dirección del Hospital Nacional y el Comité de Ética del Hospital Nacional aprobaron el protocolo de esta investigación. 


\section{RESULTADOS}

Ingresaron al estudio $76 \mathrm{RN}$, siendo 43 del sexo masculino $(56,6 \%)$ y 33 del femenino $(43,4 \%)$. La edad gestacional media fue $36 \pm 3$ semanas (rango 30 41 semanas). Las vías de parto fueron la cesárea en 52 sujetos $(68,4 \%)$ y vaginal en 24 (31,6\%). Las indicaciones de cesárea fueron: bradicardia fetal $(22,22 \%)$, sospecha dehiscencia de sutura uterina, presentación pelviana, preeclampsia y embarazo gemelar: $11,11 \%$ cada uno; otras menos frecuentes fueron: cesareada anterior, feto macrosómico, descompensación materna y Doppler fetal alterado.

La mediana del peso de los RN fue 2.569 gramos (rango intercuartílico 1.410 - 3.840 gramos).

Relacionando el peso con la edad gestacional, se encontró $39,5 \%$ adecuados a la edad gestacional, $32,9 \%$ pequeños y $27,6 \%$ grandes para la edad gestacional.

Se detectaron 42 prematuros $(55,26 \%)$. La mediana del Apgar al minuto fue 8 (rango 2-9) y a los 5 minutos fue 9 (rango 5-9).

Clasificando a los $\mathrm{RN}$ en relación al peso por percentiles, se encontró: extremo bajo peso al nacer $6,6 \%$, muy bajo peso al nacer $18,4 \%$, bajo peso al nacer $30,3 \%$, adecuado $19,7 \%$ y macrosómico $25 \%$.

Todos los RN fueron sometidos a mediciones de glicemia capilar en al menos 3 ocasiones, encontrándose gran variación en estos resultados (Tabla 1). En algunos RN se realizaron además determinaciones de glicemia plasmática (Tabla 2).

Tabla 1. Valores de glicemia capilar $(\mathrm{mg} / \mathrm{dL})$ en recién nacidos de riesgo.

\begin{tabular}{lccccc}
\hline Parámetros & $\begin{array}{c}1^{\circ} \text { control } \\
(\mathrm{n} \mathrm{76})\end{array}$ & $\begin{array}{c}2^{\circ} \text { control } \\
(\mathrm{n} 76)\end{array}$ & $\begin{array}{c}3^{\circ} \text { control } \\
\text { (n 76) }\end{array}$ & $\begin{array}{c}4^{\circ} \text { control } \\
\text { (n 52) }\end{array}$ & $\begin{array}{c}5^{\circ} \text { control } \\
\text { (n 22) }\end{array}$ \\
\hline Media $\pm \mathrm{DE}$ & $63 \pm 23$ & $82 \pm 25$ & $104 \pm 55$ & $103 \pm 37$ & $109 \pm 54$ \\
Rango & $20-137$ & $24-183$ & $39-452 *$ & $57-298$ & $57-328$ \\
\hline *valor obtenido post tratamiento con hidratación parenteral con suero glucosado
\end{tabular}

Tabla 2. Valores de glicemia plasmática $(\mathrm{mg} / \mathrm{dL})$ en recién nacidos de riesgo.

\begin{tabular}{lccccc}
\hline Parámetros & $\begin{array}{c}1^{\circ} \text { control } \\
\text { (n 26) }\end{array}$ & $\begin{array}{c}2^{\circ} \text { control } \\
\text { (n 15) }\end{array}$ & $\begin{array}{c}3^{\circ} \text { control } \\
\text { (n 11) }\end{array}$ & $\begin{array}{c}4^{\circ} \text { control } \\
\text { (n 5) }\end{array}$ & $\begin{array}{c}5^{\circ} \text { control } \\
\text { (n 2) }\end{array}$ \\
\hline Media \pm DE & $32 \pm 17$ & $45 \pm 28$ & $72 \pm 75$ & $49 \pm 241$ & $23 \pm 85$ \\
Rango & $6-63$ & $6-94$ & $2-256^{*}$ & $21-82$ & $61-269$ \\
\hline
\end{tabular}

Correlacionado el $1^{\circ}$ control de glicemia capilar con el $1^{\circ}$ control de glicemia plasmática se obtuvo una correlación considerable: $r+0,6$ (prueba correlación de Pearson) (Gráfico 1).

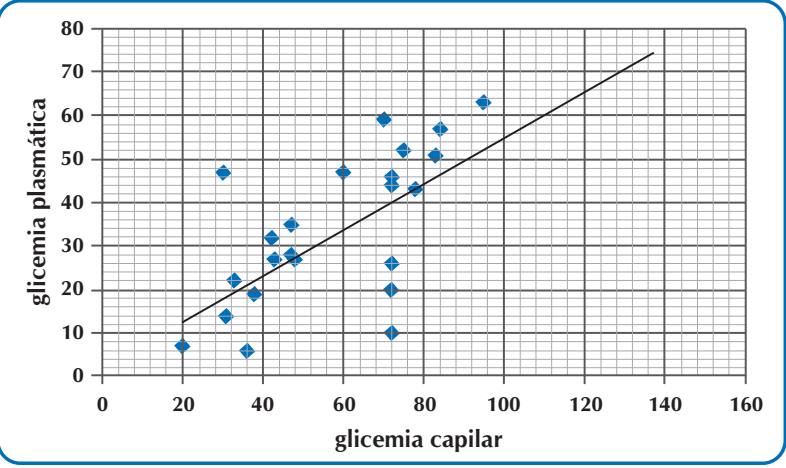

Gráfico 1. Correlación entre glicemia capilar y plasmática en recién nacidos de riesgo (n 76).

Considerando un punto de corte en la glicemia capilar en $47 \mathrm{mg} / \mathrm{dL}$ en cualquiera de los 3 primeros controles, se encontró que $25 \mathrm{RN}$ presentaron criterios para ser diagnosticados como hipoglicemia neonatal y que pasaron a conformar el grupo de casos. Actuaron como controles $51 \mathrm{RN}$ que no presentaron hipoglicemia. Determinando los factores de riesgo asociados a la hipoglicemia neonatal, se halló que la prematurez y la presencia de patologías asociadas resultaron estadísticamente significativas (Tabla 3).

Tabla 3. Factores de riesgo asociados a hipoglicemia neonatal.

\begin{tabular}{lcccc}
\hline Factores de riesgo & $\begin{array}{c}\text { Casos } \\
\text { (n 25) }\end{array}$ & $\begin{array}{c}\text { Controles } \\
\text { (n 51) }\end{array}$ & $\begin{array}{c}\text { Odds ratio } \\
\text { (IC 95\%) }\end{array}$ & Valor p* \\
\hline Patologías asociadas & $24(96 \%)$ & $25(49 \%)$ & $24(3,1-198)$ & $<0,001^{* *}$ \\
\hline Prematurez & $21(84 \%)$ & $21(41 \%)$ & $7,5(2,2-25)$ & $<0,001^{* *}$ \\
\hline RCIU & $15(60 \%)$ & $39(76 \%)$ & $2,1(0,7-6)$ & 0,1 \\
Cesárea & $16(64 \%)$ & $36(70 \%)$ & $0,7(0,2-2)$ & 0,5 \\
\hline Sexo masculino & $13(52 \%)$ & $30(58 \%)$ & $1,3(0,5-3,4)$ & 0,7 \\
Hipertensión arterial & $11(44 \%)$ & $17(33 \%)$ & $1,5(0,5-4,1)$ & 0,3 \\
\hline Diabetes mellitus & $2(8 \%)$ & $12(23 \%)$ & $0,2(0,05-1,3)$ & $0,1 * *$ \\
\hline * prueba Chi ${ }^{2}$ & $* *$ corrección Yates & &
\end{tabular}

Las patologías asociadas más frecuentes en toda la muestra fueron la dificultad respiratoria (44 RN), sepsis (15 RN) y asfixia perinatal ( $2 \mathrm{RN})$. No se pudo estimar la fuerza de asociación entre cada una de las patologías asociadas y la presencia o ausencia de hipoglicemia neonatal, pues la muestra fue insuficiente. Las causas de dificultad respiratoria fueron la enfermedad de membrana hialina $(56,8 \%)$, la taquipnea transitoria del RN (40,9\%) y el síndrome de aspiración de líquido amniótico meconial (2,3\%). 
Respecto a los casos con asfixia perinatal, un RN fue de 34 semanas, con 1800 gr y Apgar 2/6; y el segundo caso fue de 39 semanas con 4800 gr y Apgar 6/7. Ambos presentaron hipoglicemia.

Al analizar los valores de glicemia capilar en los primeros 3 controles con la alimentación enteral precoz, se halló una relación estadísticamente significativa entre hipoglicemia neonatal y la falta de dicha alimentación (Tabla 4).

Tabla 4. Relación entre glicemia capilar y alimentación enteral.

\begin{tabular}{lccc}
\hline Glicemia capilar & $\begin{array}{c}\text { Con alimentación } \\
(\mathrm{n} \mathrm{40)}\end{array}$ & $\begin{array}{c}\text { Sin alimentación } \\
(\mathrm{n} \mathrm{36)}\end{array}$ & Valor p \\
\hline $1^{\circ}$ control (mg/dL) & $72 \pm 20$ & $52 \pm 21$ & $<0,001^{*}$ \\
$2^{\circ}$ control (mg/dL) & $83 \pm 21$ & $81 \pm 29$ & $0,7^{*}$ \\
$3^{\circ}$ control (mg/dL) & $89 \pm 18$ & $119 \pm 75$ & $0,04^{* *}$ \\
\hline${ }^{*}$ prueba ANOVA & ${ }^{*}$ prueba Kruskall Wallis &
\end{tabular}

Se detectaron síntomas relacionados a la hipoglicemia en $46 \mathrm{RN}$, siendo 23 (92\%) en el grupo de casos y 23 (45\%) en el grupo de los controles. El síntoma más frecuente fue la polipnea, que se detectó en 22 casos (88\%) vs. 21 controles (42\%) $(\mathrm{p}<0,001)$. Otros síntomas, aunque muy raros, fueron la cianosis, hipotonía y letargia.

Los tratamientos realizados a los $\mathrm{RN}$ con hipoglicemia fueron la hidratación parenteral $(48 \%)$, el push de suero glucosado (13\%) y el aporte enteral (38\%).

\section{DISCUSIÓN}

En esta investigación se consideró como punto de corte para hipoglicemia neonatal a un valor de $<47$ $\mathrm{mg} / \mathrm{dL}$ teniendo en cuenta la Academia Americana de Pediatría que, a pesar que no encontró evidencia científica para discriminar entre glicemia normal y baja en el RN de pretérmino y de término, propuso como límite inferior normal esta cifra arriba mencionada ${ }^{(2,6)}$. Estudios recientes refuerzan el concepto que este valor es el límite para el desarrollo de complicaciones neurológicas a largo plazo ${ }^{(26)}$.

La mayoría de los pacientes incluidos fueron prematuros $(55,6 \%)$ lo cual es considerado uno de los factores de riesgo más importantes para desarrollar hipoglicemia. En relación al peso al nacer predominó el bajo peso al nacer y la macrosomía, que son considerados $\mathrm{RN}$ de riesgo. En el primero existe baja disponibilidad de glucosa y en los macrosómicos un mayor requerimiento ${ }^{(1,2)}$.
La media del primer control de glicemia capilar, realizado en las primeras horas de vida mostró valores normales $(63 \mathrm{mg} / \mathrm{dL})$. Como se espera, los valores de glicemia capilar en los siguientes controles evidenciaron una tendencia en ascenso. Esto se debe a los mecanismos compensadores que se ponen en marcha una vez clampado el cordón umbilical $^{(3)}$. La determinación de glicemia plasmática no es una prueba de rutina en el Servicio y no existen guías respecto a su indicación. Se realizaron algunas determinaciones y se encontró una correlación considerable $(r+0,6)$. Este hallazgo coincide con diversos artículos que mencionan la alta sensibilidad y especificidad de los glucómetros en la determinación de glicemia capilar como un método rápido y económico ${ }^{(18,19)}$.

La prematurez fue uno de los factores de riesgo detectado en esta investigación (OR 7,5 IC 95\% 2,225): coincidiendo con la mayoría de los reportes ${ }^{(1,2)}$. Este fenómeno se explica pues el hepatocito del prematuro se halla inmaduro para poner en marcha los procesos de neoglucogénesis y los depósitos de glucógeno hepáticos son escasos.

Pero el factor de riesgo de mayor valor fue la presencia de patologías asociadas (OR 24 IC 95\% 3,1198). Entre estas destacan las causas de dificultad respiratoria como la enfermedad de membrana hialina y la taquipnea transitoria del $\mathrm{RN}$; estas patologías son frecuentes en las salas de Neonatología y no se puede saber si existe una asociación causa-efecto entre las mismas y la hipoglicemia. Este tema debería investigarse a profundidad en otros estudios.

Llama la atención que los RN de madres diabéticas no presentaron alta frecuencia de hipoglicemia; esto podría deberse a que las madres con diabetes estaban mejor controladas metabólicamente ya que $93 \%$ de las mismas se encontraban compensadas ${ }^{(27)}$; reflejando el valor de los controles prenatales adecuados.

La restricción del crecimiento intrauterino fue un riesgo pero no significativo posiblemente por la baja cantidad de RN en esta muestra. Incluyendo más sujetos al estudio se podría determinar la significancia estadística.

El sexo masculino no constituyó un factor de riesgo 
significativo, al contrario como mencionan algunos autores $^{(1)}$. La hipertensión arterial materna tampoco constituyó un factor de riesgo significativo posiblemente por el mejor manejo de la presión arterial.

Se encontró una clara asociación entre la falta de alimentación enteral precoz y los niveles bajos de glicemia capilar. Esto demuestra la importancia del aporte nutricional en el metabolismo glucídico del $\mathrm{RN}^{(3)}$.

Los síntomas atribuidos clásicamente a la hipoglicemia no fueron exclusivos de los casos ya que muchos controles presentaron los mismos, sobre todo polipnea ${ }^{(6)}$. La polipnea es un síntoma común a diversas afecciones, tema que no fue objeto de esta investigación. Tampoco se investigó el desenlace clínico de los $\mathrm{RN}$ con hipoglicemia ni las eventuales secuelas.

En sujetos con hipoglicemia la hidratación parenteral fue el principal tratamiento, seguido del aporte enteral. Esta elección de tratamiento se basa en el estado clínico del paciente y la posibilidad de

\section{REFERENCIAS}

1. Van Haltren K, Malhotra A. Characteristics of infants admitted with hypoglycemia to a neonatal unit. J Pediatr Endocrinol Metab. 2013;26(5-6):525-29.

2. Committee on Fetus and Newborn, Adamkin DH. Postnatal glucose homeostasis in late-preterm and term infants. Pediatrics. 2011;127(3):575-79.

3. Mitanchez D. Glucose regulation in preterm newborn infants. Horm Res. 2007;68(6):265-71.

4. Hay WW Jr, Raju TN, Higgins RD, Kalhan SC, Devaskar SU. Knowledge gaps and research needs for understanding and treating neonatal hypoglycemia: workshop report from Eunice Kennedy Shriver National Institute of Child Health and Human Development. J Pediatr. 2009;155(5):612-17.

5. Tin W, Brunskill G, Kelly T, Fritz S. 15-year follow-up of recurrent "hypoglycemia" in preterm infants. Pediatrics. 2012;130(6): e1497-503.

6. Rozance PJ. Update on neonatal hypoglycemia. Curr Opin Endocrinol Diabetes Obes. 2014;21(1):45-50. indicar inicio de aporte enteral. El menos realizado fue el push con suero glucosado a pesar de ser considerado el tratamiento de elección en casos de hipoglicemia sintomática ${ }^{(27,28)}$. En este estudio no se evaluó la relación entre cada forma de tratamiento y el estado clínico del paciente ni la respuesta a cada tratamiento mencionado. Nuevas formas de tratamiento con gel de dextrosa $40 \%$ por vía oral aún no se aplican en nuestro Servicio aunque han demostrado su efectividad ${ }^{(29)}$.

\section{CONCLUSIONES}

Los factores de riesgo estadísticamente significativos asociados a la hipoglicemia neonatal fueron la presencia de patologías asociadas (dificultad respiratoria, sepsis y asfixia perinatal) y la prematurez. La mayoría de los $\mathrm{RN}$ de riesgo fue adecuada a la edad gestacional pero con bajo peso al nacer. La correlación entre la glicemia capilar y la plasmática fue considerable $(\mathrm{r}+0,6)$. El principal síntoma detectado en los casos de hipoglicemia fue la polipnea. La diabetes materna no se relacionó significativamente con la hipoglicemia neonatal.

7. Fernández Lorenzo JM, Couce Pico M, Fraga Bermúdez JM. Hipoglucemia neonatal. In: Asociación Española de Pediatría. Protocolos diagnóstico terapeúticos de la AEP: neonatología. España: AEP; 2008.

8. Cornblath M, Hawdon JM, Williams AF, AynsleyGreen A, Ward-Platt MP, Schwartz R, Kalhan SC. Controversies regarding definition of neonatal hypoglycemia: suggested operational thresholds. Pediatrics. 2000;105(5):1141-45.

9. Melkie M, Yigeremu M, Nigussie P, Teka T, kinde S. Is the difference in neonatal blood glucose concentration of caesarian and vaginally delivered term infants requiring separated reference intervals? BMC Res Notes. 2012;5:519.

10. Houin S, Rozance PJ. 50 years ago in the Journal of pediatrics: the incidence of neonatal hypoglycemia in a nursery for premature infants. J Pediatr. 2014;164(6):1485.

11. Nangia S. Point of care estimation of blood glucose in neonates. Indian Pediatr. 2012;49(8):612-3

12. Harris DL, Battin MR, Weston PJ, Harding JE. Continuous glucose monitoring in newborn babies at risk 
of hypoglycemia. J Pediatr. 2010;157(2):198-202.e1.

13. Ho HT, Yeung WK, Young BW. Evaluation of "point of care" devices in the measurement of low blood glucose in neonatal practice. Arch Dis Child Fetal Neonatal Ed. 2004;89(4):F356-359.

14. Hwang JH, Sohn YH, Chang SS, Kim SY. Evaluation of three glucometers for whole blood glucose measurements at the point of care in preterm or low-birth-weight infants. Korean J Pediatr. 2015;58(8):301-8.

15. Ngerncham S, Piriyanimit S, Kolatat T, Wongsiridej $P$, Inchgarm L, Kitsommart R, Vutrapongwatana P, Jeerapaet $K$. Validity of two point of care glucometers in the diagnosis of neonatal hypoglycemia. Indian Pediatr. 2012;49(8):621-25.

16. Harding JE, Hegarty JE, Crowther CA, Edlin R, Gamble G, Alsweiler JM. Randomised trial of neonatal hypoglycaemia prevention with oral dextrose gel (hPOD): study protocol. BMC Pediatr. 2015;15(1):120.

17. Senniappan $S$, Alexandrescu $S$, Tatevian N, Shah $P$, Arya V, Flanagan S, Ellard S, Rampling D, Ashworth M, Brown RE, Hussain K. Sirolimus therapy in infants with severe hyperinsulinemic hypoglycemia. N Engl J Med. 2014;370(12):1131-7.

18. Jonas D, Dietz W, Simma B. Hypoglycemia in newborn infants at risk. Klin Padiatr. 2014;226(5):287-91.

19. Rozance PJ, Hay WW Jr. Neonatal hypoglycemia-answers, but more questions. J Pediatr. 2012;161(5):775-6.

20. Thornton PS, Stanley CA, De León DD, Harris D, Haymond MW, Hussain K, Levitsky LL, Murad MH, Rozance PJ, Simmons RA, Sperling MA, Weinstein DA, White NH, Wolfsdorf JI; Pediatric Endocrine Society. Recommendations from the Pediatric Endocrine Society for Evaluation and Management of Persistent Hypoglycemia in Neonates, Infants, and Children. J Pediatr. 2015;167(2):238-45.

21. Wayenberg JL, Pardou A. Moderate hypoglycemia in the preterm infant: is it relevant? Arch Pediatr. 2008;15(2):153-56.

22. Freitas P, Matos CV, Kimura AF. Perfil de las madres de neonatos con control glucémico en las primeras horas de vida. Rev Esc Enferm USP. 2010;44(3):636-41.

23. Harris DL, Weston PJ, Harding JE. Incidence of neonatal hypoglycemia in babies identified as at risk. J Pediatr. 2012;161(5):787-91.

24. Stanley CA, Rozance PJ, Thornton PS, De León DD, Harris D, Haymond MW, Hussain K, Levitsky LL, Murad $\mathrm{MH}$, Simmons RA, Sperling MA, Weinstein DA, White $\mathrm{NH}$, Wolfsdorf JI. Re-evaluating "transitional neonatal hypoglycemia": mechanism and implications for management. J Pediatr. 2015;166(6):1520-5.e1.

25. Simmons R, Stanley C. Neonatal Hypoglycemia Studies--Is There a Sweet Story of Success Yet? N Engl J Med. 2015;373(16):1567-69.

26. McKinlay CJ, Alsweiler JM, Ansell JM, Anstice NS, Chase JG, Gamble GD, Harris DL, Jacobs RJ, Jiang Y, Paudel N, Signal M, Thompson B, Wouldes TA, Yu TY, Harding JE; CHYLD Study Group. Neonatal glycemia and neurodevelopmental outcomes at 2 Years. N Engl J Med. 2015;373(16):1507-18.

27. Yamamoto JM, Kallas-Koeman MM, Butalia S, Lodha AK, Donovan LE. Large-for-gestational-age neonate predicts a 2.5-fold increased odds of neonatal hypoglycemia in women with type 1 diabetes. Diabetes Metab Res Rev. 2017Jan;33(1). doi: 10.1002/dmrr.2824. Epub 2016Jun 21.

28. Rozance PJ, Hay WW Jr. New approaches to management of neonatal hypoglycemia. Matern Health Neonatol Perinatol. 2016;2:3.

29. Weston PJ, Harris DL, Battin M, Brown J, Hegarty JE, Harding JE. Oral dextrose gel for the treatment of hypoglycaemia in newborn infants. Cochrane Database Syst Rev. 2016 May 4;5:CD011027. 